\title{
A DEA-based approach for the multi-criteria assignment problem
}

\author{
Gholam Hassan Shirdel ${ }^{1, *}$, Ameneh Mortezaee ${ }^{2}$ \\ 1 Department of Mathematics, University of Qom, Qom, I. R. Iran \\ E-mail: 〈shirdel81math@gmail.com〉 \\ ${ }^{2}$ Department of Mathematics, University of Qom, Qom, I. R. Iran \\ E-mail: 〈ameneh-mortezaee@yahoo.com〉
}

\begin{abstract}
This paper proposes a DEA model for evaluating arc efficiency in the presence of multiple weights on arcs in a network. Thereafter, a multi-criteria assignment problem is formulated based on the efficiency of the arcs. Appraisal is given to some parts of the proposed DEA-model used for solving multi-criteria network flow problems and provide some examples. Finally, a numerical example is used to illustrate the applicability of the approach.
\end{abstract}

Key words: network flow problem, Data Envelopment Analysis, multi objective linear programming, assignment problem, efficiency.

Received: December 29, 2014; accepted: February 25, 2015; available online: March 30, 2015

DOI:10.17535/crorr.2015.0012

\section{Introduction}

One of the most applicable problems in the field of to combinatorial optimization are the network flow problems [1]. Indeed, a large set of real-world problems can be formulated as network flows. These problems are tractable when only one single weight is considered for each arc and particular algorithms in a fair computing time can be founded for solving them.

Since real-life decision making often carries a number of objectives that are to be simultaneously optimized, there are several kinds of weights on each arcs of a network in many real applications. These weights are often in conflict, and there is no solution that concurrently optimizes all objectives. Therefore, the goal is finding a solution incorporating this property such that none of the objectives can be improved without degrading the others. These solutions are called non-dominated solutions or efficient solutions.

Researchers often solve these types of problems through approaches: the weights are considered to be constraints, where the problem is then called a constrained network flow problem (CNFP), or the weights are considered to be objective functions,

${ }^{*}$ Corresponding author. 
where the problem is then called a multiple objective network flow problem, which is a particular case of MOLP (see [10, 11]).

Some researchers and mathematicians have recently presented methods for solving multi-criteria network flow problems by using the Data Envelopment Analysis technique. DEA is a mathematical programming technique, and is used to evaluate the relative efficiency of decision making units (DMU) with multiple inputs and outputs. This technique has been proposed by Charnes-Cooper-Rhodes in [8] (the CCR model) and has been extended by Banker et al, [5] (the BCC model).

In this paper, the authors have mentioned and evaluated some of the works in this field, and in addition, have propose a DEA-based method for solving the multicriteria assignment problem.

Some researchers have studied ways of solving certain multi-criteria network flow problems using DEA. In regards to the problem of assignment, Zerafat Angiz has proposed a DEA model to evaluate the efficiency of each assignment subject to nonhomogeneous costs [15]. Moreover, Chen and Lu have developed a procedure for resolving the assignment problem using multiple incommensurate inputs and outputs for each possible assignment[9]. Nonetheless, Cardillo et al. [6] and Jahanshahloo et al.[12] have presented techniques to evaluate and identify the efficient paths on a road network. Subsequently, in applying a DEA model, Hosseinzadeh et al. have determined the non-dominated paths in the multi-criteria shortest path problem (MCSPP) [14]. Finally Amirteimoori in 2007 [4] and 2012 [3] proposed methods for solving the multi-criteria transportation problem (MCTP) and the multi-criteria shortest path problem, respectively, using the DEA approach.

\section{Important concepts}

The authors have come across counterexamples in study and scrutinizing Amirteimoori's methods for solving the transportation problem and shortest path problem.

\subsection{A counterexample to Amirteimoori's method for the MCTP}

This section presents the transportation scheme given by Amirteimoori's method, which is not necessarily a non-dominated solution for extending the transportation problem as a multi objective linear programming problem.

Suppose there is a two-objective transportation problem on the network $\mathbb{G}=$ $(\mathbb{N}, \mathbb{A})$ with two warehouses and three destinations. The variable $\left(c_{i j}, p_{i j}\right)$ is the (cost,profit) on arc $(i, j)$ for transportation of a unit commodity on this arc, as shown in Table (1). The aim is to send the commodity from a warehouse to destination for supply and demand purposes, so that the objective functions to become efficient. By solving this MOLP, the efficient solution is $x_{11}^{*}=x_{13}^{*}=15, x_{22}{ }^{*}=x_{23}^{*}=10$ and $x_{12}^{*}=x_{21}^{*}=0$ (where $x_{i j}$ means that it can be transport the $x_{i j}$ unit commodity along arc $(i, j))$. The objective vector of this solution is $Z^{*}=(205,460)$.

To determine an efficient transportation plan with maximum efficiency utilizing the method proposed by Amirteimoori [4], the following model based on the data in Table (2) is solved. 


\begin{tabular}{|l|c|c|c|c|}
\hline & destination 1 & destination 2 & destination 3 & $\mathrm{~s}_{i}$ \\
\hline warehouse 1 & $(4,10)$ & $(7,6)$ & $(5,10)$ & 30 \\
\hline warehouse 2 & $(2,5)$ & $(4,6)$ & $(3,10)$ & 20 \\
\hline $\mathrm{d}_{j}$ & 15 & 10 & 25 & \\
\hline
\end{tabular}

Table 1: A transportation problem with two objectives

\begin{tabular}{|l|c|c|c|c|c|}
\hline Arc & $\mathrm{c}_{i j}$ & $\mathrm{p}_{i j}$ & $\mathrm{e}_{i j}{ }^{1}$ & $\mathrm{e}_{i j}{ }^{2}$ & $\mathrm{e}_{i j}$ \\
\hline$(1,1)$ & 4 & 10 & 1 & 1 & 1 \\
\hline$(1,2)$ & 7 & 6 & $12 / 35$ & $4 / 7$ & $16 / 35$ \\
\hline$(1,3)$ & 5 & 10 & $4 / 5$ & $3 / 5$ & $7 / 10$ \\
\hline$(2,1)$ & 2 & 5 & $3 / 4$ & 1 & $7 / 8$ \\
\hline$(2,2)$ & 4 & 6 & $9 / 20$ & 1 & $29 / 40$ \\
\hline$(2,3)$ & 3 & 10 & 1 & 1 & 1 \\
\hline
\end{tabular}

Table 2: Data and results

$$
\begin{aligned}
\min & \sum_{(i, j) \in A}\left(1-e_{i j}\right) x_{i j} \\
\text { s.t. } & \sum_{j=1}^{n} x_{i j}=s_{i}, \quad i=1, \ldots, m \\
\sum_{i=1}^{m} x_{i j}=d_{j}, & j=1, \ldots, n \\
x_{i j} \geq 0, & \forall(i, j) \in A .
\end{aligned}
$$

The efficient transportation plan with maximum efficiency upon solving model (2.1) is $x_{11}=15, x_{12}=10, x_{13}=5, x_{23}=20, x_{12}=x_{22}=0$. The cost and profit corresponding to this solution is $Z_{X}=(215,460)$.

It becomes obvious that $Z_{X} \geq Z^{*}$. Consequently $X$ is not a non-dominated (efficient) solution to this problem.

\subsection{A counterexample to Amirteimoori's method for MCSPP}

Suppose that the directed network $\mathbb{G}=(\mathbb{N}, \mathbb{A})$ with five nodes and eight arcs. In this network, a cost and a profit $\left(c_{i j}, p_{i j}\right)$ are considered on each arc $(i, j)$, as shown in Figure (1). It becomes obvious that the path $p=(1 \rightarrow 4 \rightarrow 5)$ is the unique non-dominated (or efficient) path from node 1 to node 5 with an objective vector $(4,11)$. 


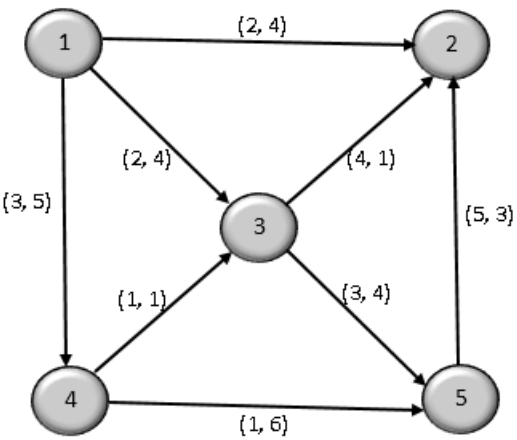

Figure 1: Network $\mathbb{G}=(\mathbb{N}, \mathbb{A})$ with two objective functions

To determine an efficient path from node 1 to node 5 with maximum efficiency using the method proposed by Amirteimoori [3], the model (2.2) is solved using data from Table (3).

\begin{tabular}{|c|c|c|c|c|c|}
\hline Arc & $\mathrm{c}_{i j}$ & $\mathrm{p}_{i j}$ & $\mathrm{e}_{i j}{ }^{1}$ & $\mathrm{e}_{i j}{ }^{2}$ & $\mathrm{e}_{i j}$ \\
\hline$(1,2)$ & 2 & 4 & 1 & 1 & 1 \\
\hline$(1,3)$ & 2 & 4 & 1 & 1 & 1 \\
\hline$(1,4)$ & 3 & 5 & $5 / 6$ & 1 & $11 / 12$ \\
\hline$(2,5)$ & 5 & 3 & 1 & $1 / 10$ & $11 / 20$ \\
\hline$(3,2)$ & 4 & 1 & $3 / 16$ & $1 / 8$ & $5 / 32$ \\
\hline$(3,5)$ & 3 & 4 & 1 & $2 / 9$ & $11 / 18$ \\
\hline$(4,3)$ & 1 & 1 & $1 / 6$ & $1 / 2$ & $1 / 3$ \\
\hline$(4,5)$ & 1 & 6 & 1 & 1 & 1 \\
\hline
\end{tabular}

Table 3: Data and efficiency of each arcs

$$
\begin{array}{cl}
\max \sum_{(i, j) \in A} e_{i j} x_{i j} & \\
\text { s.t. } \sum_{j:(i, j) \in A} x_{i j}-\sum_{j:(j, i) \in A} x_{j i}= \begin{cases}1 & \text { if } i=1 \\
0 & \text { if } i \neq 1 \text { or } i \neq n \\
-1 & \text { if } i=n\end{cases} \\
x_{i j} \in\{0,1\}, \quad \forall(i, j) \in A .
\end{array}
$$

The efficient path with a maximum efficiency achieved by solving model (1) is the path

$q=(1 \rightarrow 4 \rightarrow 3 \rightarrow 2 \rightarrow 5)$. The cost and profit correspond to this path is $(13,10)$.

It is obvious that path $\mathrm{p}$ dominates path $\mathrm{q}$. Consequently, path $\mathrm{q}$ is not an efficient path. 
Therefore, it seems that the efficiency that Amirteimoori defines, for each arc in an extended shortest path problem and in an extended transportation problem, is not a suitable criterion for evaluating the measure of efficiency of solutions to these problems and in each case, it leads to dominated solution.

In this work, a DEA model is proposed for evaluating arc efficiency in a network. Here, in regard to the multi-criteria assignment problem that will be described in section (4), each assignment(in other words, each arc) can be considered a decision making unit by replacing its weights that are to be minimized with inputs(costs) and its weights to be maximized with outputs(profits).Thus, there are several DMUs with multiple inputs and multiple outputs. This assignment is then evaluated using other assignments in the same row.

The next section DEA is introduces. In section (4) an assignment problem is studied, and in Section 5, the authors propose a method for finding an efficient solution to the multi-criteria assignment problem utilizing DEA. In section 6, an example illustrates the procedure. Finally, 7 presents the conclusion.

\section{Data Envelopment Analysis}

DEA is a mathematical programming technique that has been proposed by Charnes et al. [8]. The technique has been extended by Banker et al. [5]. The additive model which is used in this paper, has been proposed by Charnes et al.[7].

Suppose there are $\mathrm{n}$ decision making units, $D M U_{j}$, for $j=1,2, \cdots, n$, where each $D M U_{j}$ consumes a m-vector input, $X_{j}=\left(x_{1 j}, \cdots, x_{m j}\right)^{T}$, to produce an s-vector output, $Y_{j}=\left(y_{1 j}, \cdots, y_{s j}\right)^{T}$.

For evaluating relative efficiency of $D M U_{p}$, the additive model is used and is as follows:

$$
\begin{aligned}
& \min h_{p}=-\sum_{i=1}^{m} s_{i}^{-}-\sum_{r=1}^{s} s_{r}^{+} \\
& \text {s.t } \quad-\sum_{j=1}^{n} \lambda_{j} x_{i j}-s_{i}^{-}=-x_{i p} \quad i=1, \cdots, m \\
& \sum_{j=1}^{n} \lambda_{j} y_{r j}-s_{r}^{+}=y_{r p} \quad r=1, \cdots, s \\
& \sum_{j=1}^{n} \lambda_{j}=1 \\
& \lambda_{j} \geq 0 \\
& j=1, \ldots, n \\
& s_{i}^{-} \geq 0 \\
& i=1, \ldots, m \\
& s_{r}^{+} \geq 0 \\
& r=1, \ldots, s
\end{aligned}
$$


Where $s_{i}^{-}$and $s_{r}^{+}$are the slack variables of the corresponding constraints of the $i^{\text {th }}$ input and $r^{\text {th }}$ output, respectively. The dual of model (3.1) is:

$$
\begin{aligned}
& \max g_{p}=\sum_{r=1}^{s} u_{r p} y_{r p}+\sum_{i=1}^{m} v_{i p} x_{i p}+u_{0 p} \\
& \text { s.t. } \quad \sum_{r=1}^{s} u_{r p} y_{r j}-\sum_{i=1}^{m} v_{i p} x_{i j}+u_{0 p} \leq 0 \quad j=1, \ldots, n \\
& -u_{r p} \leq-1 \quad r=1, \ldots, s \\
& -v_{i p} \leq-1 \quad i=1, \ldots, m
\end{aligned}
$$

$\mathrm{DMU}_{p}$ is efficient in the additive model if and only if $h_{p}^{*}=g_{p}^{*}=0$.

Lemma 1. The additive model is translation invariant.

Proof. For more details, see [2].

Since the additive model is translation invariant, it can be used it for evaluating DMUs with a zero or negative component of the input/output vector.

\section{Multi-criteria assignment problem}

The assignment problem is a special case of the transportation problem. In this case, each source supplies only one commodity and each destination is applicable for only one commodity. One of the popular methods for solving this problem is the Hungarian method which was presented in 1955 by Hungarian mathematician Kuhn [13].

The standard mathematical model for the assignment problem is given by:

$$
\begin{aligned}
& \min \sum_{i=1}^{n} \sum_{j=1}^{n} c_{i j} x_{i j} \\
& \text { s.t } \quad \sum_{i=1}^{n} x_{i j}=1 \quad j=1,2, \ldots, n \\
& \sum_{j=1}^{n} x_{i j}=1 \quad i=1,2, \ldots, n \\
& x_{i j} \in\{0,1\} \quad i, j=1,2, \ldots, n
\end{aligned}
$$

As mentioned above, only one $\operatorname{cost}\left(c_{i j}\right)$ is considered in the above model for each assignment, but in many real-life problems it may be that any assignment include several non-homogenous costs(or profits). Therefore, a cost vector $C_{i j}=$ $\left(c_{i j}^{1}, c_{i j}^{2}, \cdots, c_{i j}^{m}\right)$ and a profit vector $P_{i j}=\left(p_{i j}^{1}, p_{i j}^{2}, \cdots, p_{i j}^{s}\right)$ corresponding to each assignment(see Table 4). Thus, a multi-criteria assignment problem is encountered which is a special case of MOLP. 


\begin{tabular}{c|ccccc} 
& $P_{1}$ & $\cdots$ & $P_{j}$ & $\cdots$ & $P_{n}$ \\
\hline$M_{1}$ & $\left(C_{11}, P_{11}\right)$ & $\cdots$ & $\left(C_{1 j}, P_{1 j}\right)$ & $\cdots$ & $\left(C_{1 n}, P_{1 n}\right)$ \\
$\vdots$ & & & & & \\
$M_{i}$ & $\left(C_{i 1}, P_{i 1}\right)$ & $\cdots$ & $\left(C_{i j}, P_{i j}\right)$ & $\cdots$ & $\left(C_{i n}, P_{i n}\right)$ \\
$\vdots$ & & & & & \\
$M_{n}$ & $\left(C_{n 1}, P_{n 1}\right)$ & $\ldots$ & $\left(C_{n j}, P_{n j}\right)$ & $\cdots$ & $\left(C_{n n}, P_{n n}\right)$
\end{tabular}

Table 4: An assignment problem with non-homogenous weights

\section{The proposed method}

In this section, the multi-criteria assignment problem and its relative table(Table 4) is considered. Each square in this table is considered a $\operatorname{DMU}_{i j}(i, j=1,2, \cdots, n)$ with input vector $C_{i j}$ and output vector $P_{i j}$. To evaluate the $\mathrm{DMU}_{i j}$, selections of person $\mathrm{i}$ are evaluated for the purpose of implementing different projects in a combined manner. In other words, each $\mathrm{DMU}_{i j}$ is evaluated with all other DMUs found in the same row with it. The obtained outcomes of these evaluations are considered a criterion for testing the assignment in terms of viewpoint amount cost and profit values.

This evaluation is performed by utilizing the additive model. Accordingly, if $\mathrm{DMU}_{i j}$ is in row $\mathrm{i}$ and column $\mathrm{j}$ of the assignment table then the following model is solved for the purpose of evaluating this DMU in comparison with other elements of row i.

$$
\begin{array}{rlr}
s_{i j}=\max & \sum_{r=1}^{s} u_{r} p_{i j}^{r}-\sum_{k=1}^{m} v_{k} c_{i j}^{k}+u_{0} & \\
\text { s.t } & \sum_{r=1}^{s} u_{r} p_{i l}^{r}-\sum_{k=1}^{m} v_{k} c_{i l}^{k}+u_{0} \leq 0 & l=1,2, \ldots, n \\
& -v_{k} \leq-1 & \\
& -u_{r} \leq-1 & k=1,2, \ldots, m \\
& & r=1,2, \ldots, s
\end{array}
$$

$D M U_{i j}$ is strongly efficient if $s_{i j}=0$. In other words, all strongly-efficient assignments in each row are determined using the above additive model. It should be noted that when the value of $s_{i j}$ is smaller, in other words, has a larger absolute value, it indicates that assignment of project $\mathrm{j}$ with respect to person $\mathrm{i}$, when compared with assignment of other projects with respect to person i, is not suitable. Thus, $-s_{i j}$ can be used as a benchmark for evaluating this assignment. In other words, $-s_{i j}$ can be considered a combinatorial cost of assignment project $\mathrm{j}$ to person $\mathrm{i}$ in classic assignment problem, and the following model for an assignment scheme with 
minimal combinatorial cost is deemed solved.

$$
\begin{array}{lll}
\min & \sum_{i=1}^{n} \sum_{j=1}^{n}-s_{i j} x_{i j} & \\
\text { s.t } & \sum_{i=1}^{n} x_{i j}=1 & j=1,2, \ldots, n \\
& \sum_{j=1}^{n} x_{i j}=1 & i=1,2, \ldots, n \\
& x_{i j} \in\{0,1\} & i, j=1,2, \ldots, n
\end{array}
$$

\subsection{Scrutiny of the proposed method}

There are two important points presented in this method. First, since the additive model is translation invariant, this method can be performed for any variant of problem. Chen's method and Zerafatangiz's method are applicable only for problems with a positive cast (profit)-vector, because they use the CCR and BCC models. Second, in solving additive models, efficient units are determined in row $i(i=$ $1,2, \cdots, n)$ and for this unit, the combinatorial cost is zero. In other words, these units are the best assignment for person $i$. Moreover, for other assignments in row $i$, the sum of the total surplus cost and deficit profit of the related assignment is obtained, a reference to DMU in the row and then we consider it as combinatorial cost of it assignment. It is clear that if the absolute value of this number is smaller, the respective assignment is more suitable (Note that the combinatorial cost of efficient assignment in each row equals zero). Therefore, based on what has been said, the conclusion is that the optimum solution of model (5.2) is a non-dominated solution for the given problem. In addition, the value number calculated for the method is much less than the that calculated in Chen's method and Zerafatangiz's method. To verify this claim, Chen's method solves two models corresponding to each assignment. Furthermore, Zerafatangiz's method is used to solve one model with $n^{2}$ constraints corresponding to each assignment, while the proposed method solves one model with $n$ constraints corresponding to each assignment.

\section{Example}

Suppose assignment Table (5) has two non-homogenous costs corresponding to each assignment. After solving the model (5.1) for each row and obtaining $s_{i j}$ for $i, j=$ $1,2,3$, assignment Table (6) with combinatorial cost $-s_{i j}$ is obtained. Now, using the data from Table (6) and the Hungarian method, the optimum solution is $x_{11}^{*}=$ $x_{23}^{*}=x_{32}^{*}=1$ with a cost vector $(8,8)$. It becomes obvious that the obtained solution is one of the efficient assignment schemes for this problem. 


\begin{tabular}{|l|l|l|}
\hline$(3,2)$ & $(5,3)$ & $(7,6)$ \\
\hline$(4,1)$ & $(6,2)$ & $(2,5)$ \\
\hline$(8,4)$ & $(3,1)$ & $(9,1)$ \\
\hline
\end{tabular}

Table 5: Assignment table with two costs

\begin{tabular}{|l|l|l|}
\hline 0 & 3 & 8 \\
\hline 0 & 3 & 0 \\
\hline 8 & 0 & 6 \\
\hline
\end{tabular}

Table 6: Assignment table with a combinatorial cost

\section{Conclusion}

This paper, discusses the most practical groups of linear programming applied to multiple objective network flow problems. A DEA-based approach is proposed for solving the special case of, the multiple objective assignment problem. It is evident that each DEA problem can be considered a MOLP and the efficient DMUs determined. However, each MOLP can not be solved by DEA methods in general. This paper has endeavored to solve a special case of MOLP using DEA. Perhaps in future there suggestions contain herein, can be applied to the use of DEA methods for solving other kind of MOLP-related problems.

\section{References}

[1] Ahuja, R.K., Magnanti, T.L. and Orlin, J. (1993). Network Flows: Theory, Algorithms, and Applications. Upper Saddle River, USA, Prentice Hall.

[2] Ali, I. and Seiford, L. (1990). Translation invariance in Data Envelopment Analysis. Operations Research Letters, 9(6), 403-405. doi:10.1016/0167-6377(90)90061-9.

[3] Amirteimoori, A. (2012). An extended shortest path problem: A Data Envelopment Analysis approach. Applied Mathematics Letters. 25(11), 1839-1843. doi:10.1016/j.aml.2012.02.042.

[4] Amirteimoori, A. (2011). An extended transportation problem: A DEA-based approach. Central European Journal of Operations Research, 19(4), 513-521. doi:10.1007/s10100-010-0140-0.

[5] Banker, R.D., Charnes, A. and Cooper, W.W. (1984). Some models for estimating technical and scale efficiencies in Data Envelopment Analysis. Manage. Sci., 30(9), 1078-1092. doi:10.1287/mnsc.30.9.1078.

[6] Cardillo, D.D.L. and Fortuna, T. (2000). A DEA model for the efficiency evaluation of nondominated paths on a road network. European J. Oper. Res., 121(3), 549-558. doi:10.1016/s0377-2217(99)00053-3.

[7] Charnes, A., Cooper, W.W., Golany, B., Seiford, L. and Stutz, J. (1985). Foundations of Data Envelopment Analysis and Pareto-koopmans empirical production function. Journal of Econometrics, 30, 91-107. doi:10.1016/0304-4076(85)90133-2.

[8] Charnes, A., Cooper, W.W. and Rhodes, E. (1978). Measuring the efficiency of decision making units. European J. Oper. Res., 2(6), 429-444. doi:10.1016/03772217(78)90138-8.

[9] Chen, L.H. and Lu, H.W. (2007). An extended assignment problem considering multiple inputs and outputs. Appl. Math. Model., 3, 2239-2248. doi:10.1016/j.apm.2006.08.018. 
[10] Figueira,J., Greco,S. and Ehrgott, M. (2005). Multiple Criteria Decision Analysis: State of the Art Surveys. New York, Volume 78 of International Series in Operations Research Management Science. Springer Science + Business Media, Inc.

[11] Hwang, C.L. and Masud, A.S.M. (1979). Multiple Objective Decision-Making: Methods and Applications. Heidelberg, Springer-Verlag.

[12] Jahanshahloo, G.R., Hosseinzadeh Lotfi, F., Shoja, N., Tohidi, G. and Razavyan, S. (2005). The identification of nondominated and efficient paths on a network. Applied Mathematics and Computation, 169, 866-873. doi:10.1016/j.amc.2004.09.067.

[13] Kuhn, H.W. (1955). The Hungarian method for the assignment problem. Naval Res. Logist. Quart., 2, 83-97. doi:10.1002/nav.3800020109.

[14] Masoumi, M.M., Hosseinzadeh Lotfi, F. and Mobasseri, A.M. (2010). DEA and muliobjective shortest path problems. Contemporary Engineering Sciences, 3(2), 53-62.

[15] Zarafat Angiz, M., Saati, S. and Mokhtaran, M. (2003). An alternative approach to assignment problem with non-homogeneous costs using common set of weights in DEA. Far East J. Appl. Math., 10(1), 29-39. 